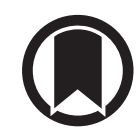

CrossMark

\title{
Neutrophil proteases alter the interleukin-22-receptor-dependent lung antimicrobial defence
}

\author{
Antoine Guillon ${ }^{1,2,3}$, Youenn Jouan ${ }^{1,2,3}$, Deborah Brea, ${ }^{1,2}$, Fabien Gueugnon ${ }^{1,2}$, \\ Emilie Dalloneau $^{1,2}$, Thomas Baranek ${ }^{1,2}$, Clémence Henry ${ }^{1,2}$, Eric Morello ${ }^{1,2}$, \\ Jean-Christophe Renauld ${ }^{4,5}$, Muriel Pichavant ${ }^{6,7,8,9}$, Philippe Gosset ${ }^{6,7,8,9}$, \\ Yves Courty ${ }^{1,2}$, Patrice Diot ${ }^{1,2}$ and Mustapha Si-Tahar ${ }^{1,2}$
}

\begin{abstract}
Affiliations: ${ }^{1}$ INSERM, Centre d'Etude des Pathologies Respiratoires, U1100, Tours, France. ${ }^{2}$ Université François Rabelais de Tours, Tours, France. ${ }^{3}$ Service de Réanimation Polyvalente, Centre Hospitalier Régional Universitaire de Tours, Tours, France. ${ }^{4}$ Ludwig Institute for Cancer Research, Brussels, Belgium. ${ }^{5}$ De Duve Institute, Universite Catholique de Louvain, Brussels, Belgium. ${ }^{6}$ Université Lille Nord de France, Lille, France. ${ }^{7}$ Lille Centre for Infection and Immunity, Institut Pasteur de Lille, Lille, France. ${ }^{8}$ Unité Mixte de Recherche 8204, Centre National de la Recherche Scientifique, Lille, France. ${ }^{9}$ INSERM, U1019, Team 8, Lille, France.
\end{abstract}

Correspondence: Mustapha Si-Tahar, Centre d’Etude des Pathologies Respiratoires, INSERM U1100, Faculté de Médecine - 10, Boulevard Tonnellé, 37032 Tours, France. E-mail: si-tahar@univ-tours.fr

ABSTRACT Chronic obstructive pulmonary disease (COPD) is punctuated by episodes of infectiondriven acute exacerbations. Despite the life-threatening nature of these exacerbations, the underlying mechanisms remain unclear, although a high number of neutrophils in the lungs of COPD patients is known to correlate with poor prognosis. Interleukin (IL)-22 is a cytokine that plays a pivotal role in lung antimicrobial defence and tissue protection. We hypothesised that neutrophils secrete proteases that may have adverse effects in COPD, by altering the IL-22 receptor (IL-22R)-dependent signalling.

Using in vitro and in vivo approaches as well as reverse transcriptase quantitative PCR, flow cytometry and/or Western blotting techniques, we first showed that pathogens such as the influenza virus promote $I L-22 R$ expression in human bronchial epithelial cells, whereas Pseudomonas aeruginosa, bacterial lipopolysaccharide or cigarette smoke do not. Most importantly, neutrophil proteases cleave IL-22R and impair IL-22-dependent immune signalling and expression of antimicrobial effectors such as $\beta$-defensin- 2 . This proteolysis resulted in the release of a soluble fragment of IL-22R, which was detectable both in cellular and animal models as well as in sputa from COPD patients with acute exacerbations.

Hence, our study reveals an unsuspected regulation by the proteolytic action of neutrophil enzymes of IL-22-dependent lung host response. This process probably enhances pathogen replication, and ultimately COPD exacerbations.

@ERSpublications

Neutrophil proteases alter lung antimicrobial defence and may predispose to infection-triggered COPD exacerbations http://ow.ly/MHqEt

Received: Nov 212014 | Accepted after revision: April 202015 | First published online: Aug 062015

This article has supplementary material available from erj.ersjournals.com

The study was approved by the French national bioethics authorities (CPP-37 2012-R21)

Support statement: A. Guillon was funded by an INSERM clinical fellowship and E. Morello was funded by an INSERM postdoctoral fellowship. E. Dalloneau was funded by Vaincre la Mucoviscidose (Defeating Cystic Fibrosis) and C. Henry and Y. Jouan were funded by Fonds de Dotation Recherche en Santé Respiratoire (Research Endowment Fund in Respiratory Health). These funding agencies had no role in study design, the collection and analysis of data, the decision to publish or the preparation of the manuscript. Funding information for this article has been deposited with FundRef.

Conflict of interest: Disclosures can be found alongside the online version of this article at erj.ersjournals.com

Copyright OERS 2015 


\section{Introduction}

Acute episodes of exacerbation mark the progression of chronic obstructive pulmonary disease (COPD), leading to substantial morbidity and mortality. COPD exacerbations are triggered mostly by respiratory viruses and bacteria, which infect the lower airway and promote persistent airway inflammation [1]. The mechanisms responsible for the increased susceptibility of COPD patients to respiratory pathogens are still unclear.

Interestingly, several studies have shown that interleukin (IL)-22, through its receptor (IL-22R), promotes antimicrobial immunity, inflammation and tissue repair at barrier surfaces [2, 3]. IL-22 is part of the IL-10 cytokine family. The cell surface IL-22R complex consists of the receptor chains IL-22R1 and IL-10R2. IL-22 is an unusual interleukin because it does not directly regulate the function of immune cells and instead targets cells at barriers that separate the body from its external environment, such as the respiratory epithelium. We and others previously demonstrated that IL-22 protects and regenerates respiratory epithelial cells upon infection with influenza virus and limits secondary bacterial infections [4]. COPD is also characterised by high numbers of leukocytes, such as neutrophils in the lungs $[5,6]$. The accumulation and activation of neutrophils in COPD result in the excessive secretion of inflammatory molecules. Many studies suggest that neutrophil-derived proteases such as elastase and cathepsin $\mathrm{G}$ are key mediators of lung damage [5-7]. Neutrophil proteases degrade soluble mediators or matrix components and alter cell surface receptors [5]. Thus, we and others previously demonstrated that elastase and cathepsin G can deactivate receptors involved in host defence and inflammation, including the lipopolysaccharide (LPS) co-receptor CD14 [8,9] and various protease-activated receptors [10, 11].

Defects in innate defence mechanisms have been documented in patients suffering from COPD, despite the abundance of innate immune cells such as neutrophils, which predominate in the airway wall and lumen of COPD patients $[5,6]$. Such immune defects may predispose COPD patients to viral or bacterial infections, which aggravate the disease.

Regarding the IL-22/IL-22R antimicrobial pathway, we hypothesised that any alteration of IL-22R in the lungs of COPD patients may compromise innate defence mechanisms and enhance susceptibility to infections. More specifically, we examined whether IL-22R expressed on lung epithelial cells is targeted by the neutrophil proteases present at the surface of lung mucosa. Our current findings establish for the first time that neutrophil serine proteases cleave the IL-22R1 receptor subunit and inhibit IL-22-mediated epithelial cell responses, such as the production of antimicrobial peptides.

Methods

Detailed methods are available in the online supplementary material.

Virus, bacteria, cell cultures and mice

The pathogenic human-origin H3N2 Influenza A virus (IAV) strain Scotland/20/74 and the Pseudomonas aeruginosa mutant strain PAK $\triangle p s c F$ have been described previously $[12,13]$. Experiments were performed using the human bronchial epithelial cell line BEAS-2B and primary human cell cultures (MucilAir; Epithelix Sarl, Geneva, Switzerland). Protocols involving BALB/c mice (female, 18-20 g) were approved by the local ethics committee (agreement CEEA VdL 2012-12-6).

\section{Preparation of cigarette smoke extract}

The smoke from two cigarettes was bubbled into $10 \mathrm{~mL}$ of medium and the resulting cigarette smoke extract (CSE) solution was considered to be $100 \%$ CSE. Control medium was made by bubbling room air into medium under the same conditions.

\section{Neutrophil activation, measurement and inhibition of protease activities}

Human blood neutrophils were purified as described previously [14]. Proteinase activity was measured as described in [15], and specific fluorescence resonance energy transfer substrates of neutrophil elastase $(\mathrm{NE})$ or cathepsin $\mathrm{G}(\mathrm{CG})$ were used.

\section{Infection and treatment of lung epithelial cells}

Cells were incubated with the following microbial triggers to investigate the effects of infection: IAV at a multiplicity of infection (MOI) of 1 , polyinosinic:polycytidylic acid (poly(I:C)) at $5 \mu \mathrm{g} \cdot \mathrm{mL}^{-1}$, LPS at $10 \mu \mathrm{g} \cdot \mathrm{mL}^{-1}$ or $P$. aeruginosa a a MOI of 1 . Cells were also exposed to $5 \%$ CSE or $5 \%$ room air media (made from freshly prepared $100 \%$ CSE or room air media) for $6 \mathrm{~h}$ to investigate the effects of CSE. Finally, cells were incubated with either supernatant from activated neutrophils, NE or CG for $30 \mathrm{~min}$ at $37^{\circ} \mathrm{C}$ to investigate the effects of neutrophil serine proteases. The cells were then washed with PBS and reactions were stopped by the addition of a protease inhibitor cocktail. 
Infection and treatment of mice

CSE-challenged mice

Animals were exposed to the smoke of 18 cigarettes twice daily ( 5 days.week ${ }^{-1}$ ) for $8-16$ weeks using a whole-body smoke exposure system. Age-matched control animals were exposed to room air only.

Mice infected with IAV

Mice were infected intranasally with IAV in sterile PBS in a total volume of $40 \mu \mathrm{L}$. For sublethal infection, $3 \times 10^{3}$ plaque-forming units of $\mathrm{H} 3 \mathrm{~N} 2 \mathrm{IAV}$ were instilled.

\section{CG-challenged mice}

CG, $\alpha$-antichymotrypsin (ACT)-treated CG or PBS alone was administered intranasally to the mice in a final volume of $40 \mu \mathrm{L}$. Bronchoalveolar lavage (BAL) was performed $2 \mathrm{~h}$ after instillation.

\section{Human samples}

Human lung tissues from nonsmokers, smokers and COPD patients were obtained from patients undergoing surgery for lung carcinoma. Lung samples were located $\geqslant 3 \mathrm{~cm}$ away from the edge of the tumour, and the absence of carcinoma was verified histologically. Sputa were collected prospectively from COPD patients or patients with acute exacerbation (AE) of COPD, and the presence of IL-22R1 receptor was assessed by Western blotting. A pool of sputum served as calibrator for comparisons. The study was approved by the French national bioethics authorities (CPP-37 2012-R21). Informed written consent was obtained from each participant.

\section{Statistical analysis}

Results are expressed as mean \pm SEM. Statistical significance was analysed using the Mann-Whitney test or the Kruskal-Wallis test (and Dunn's test for post hoc comparisons) according to the number of groups to be analysed. Statistical analysis was performed using GraphPad Prism 5 (La Jolla, CA, USA). A p-value $<0.05$ was considered significant.

\section{Results}

Viral infection upregulates IL-22R in lung epithelial cells

IL-22/IL-22R1 stimulates epithelial host defence and maintains epithelial homeostasis [2]. However, it is not known how microbial triggers regulate $I L-22 R 1$ expression in lung epithelial cells. This prompted us to assess the expression of $I L-22 R 1$ using reverse transcriptase quantitative PCR and flow cytometry in human bronchial epithelial cells exposed or not to 1) replicative IAV; 2) poly(I:C), a synthetic viral mimetic agonist; 3) the Gram-negative bacteria P. aeruginosa; or 4) LPS, a major cell wall component of Gram-negative bacteria. IL-22R1 was constitutively expressed in human bronchial epithelial BEAS-2B cells. $I L-22 R 1$ expression was higher in IAV-infected cells and poly(I:C)-challenged cells than in control cells (8.6- and 6.1-fold higher, respectively, $\mathrm{p}<0.01$; fig. 1a). By contrast, neither $P$. aeruginosa nor LPS affected the expression of $I L-22 R 1$ (fig. 1a). These results were confirmed at the protein level using flow cytometry (fig. 1b). This lack of modulation was not due to a difference in the immunostimulatory activity between bacterial and viral triggers, because the secretion of IL- 6 upon exposure of BEAS-2B cells to each stimulus was very similar (>1000 pg.mL $\mathrm{mL}^{-1}$; fig. 1c). In vivo, $I L-22 R 1$ was approximately 200 -fold higher in mouse upper airways (tracheal and bronchial compartment) than in the distal lung compartment $(\mathrm{p}<0.0001$; data not shown). IL-22R1 was also significantly upregulated in murine airways infected by IAV (3.1-fold higher than in control mice, $\mathrm{p}<0.01$; fig. $1 \mathrm{~d}$ ).

\section{Cigarette smoke does not modulate IL-22R expression}

Recurrent infections in smokers or COPD patients suggest that these individuals have defects in innate immunity defence mechanisms against lung pathogens [16]. We investigated whether IL-22R1 expression was impaired by cigarette smoke in epithelial cells, because these cells are the first to come into contact with inhaled smoke. As expected, epithelial cells exposed to CSE produced high levels of IL-8 (3.6-fold higher, $\mathrm{p}<0.01)$ and $\mathrm{NAD}(\mathrm{P}) \mathrm{H}$ quinone oxidoreductase $1(2.6$-fold higher, $\mathrm{p}<0.05)$ than in control cells (not shown). However, IL-22R1 mRNA and protein expression was unaffected by exposure to CSE ( $\mathrm{p}>0.05$; fig. 2a).

The exposure of bronchial epithelial cells to CSE is a reductionist and acute experimental model; therefore, we also verified these findings in mice chronically exposed to cigarette smoke for 8-16 weeks. Survival was not affected by smoke exposure. As expected, induction of emphysema (as assessed by alveolar space enlargement) was observed and CXCL1 expression was significantly upregulated (fig. 2b). By contrast, smoke exposure did not affect the abundance of $I L-22 R 1$ RNA in the lungs ( $>0.05$; fig. 2b). Nevertheless, we subsequently analysed $I L-22 R 1$ expression in the lungs of COPD patients. Indeed, COPD is a 

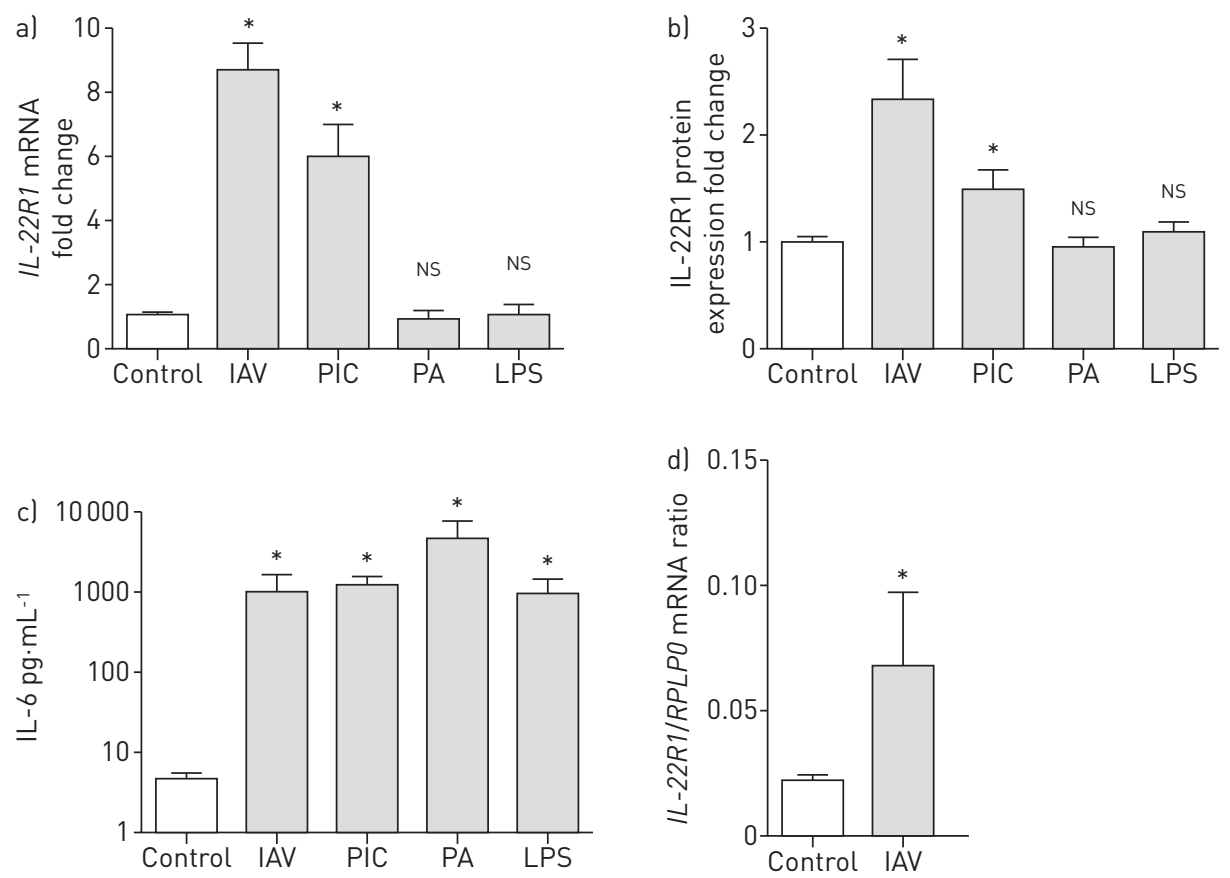

FIGURE 1 Microbial triggers regulate interleukin (IL)-22 receptor (IL-22R) expression. Human bronchial epithelial BEAS-2B cells were either infected with influenza A virus (IAV) at multiplicity of infection (MOI) $=1$ for $20 \mathrm{~h}$; treated with polyinosinic:polycytidylic acid (PIC) at $5 \mu \mathrm{g} \cdot \mathrm{mL}^{-1}$ for $20 \mathrm{~h}$; infected with Pseudomonas aeruginosa mutant strain PAK $\triangle p s c F(\mathrm{PA})$ at MOI=1 for $4 \mathrm{~h}$ (bacteria were then removed and medium with gentamycin was added for $16 \mathrm{~h}$ ); or treated with lipopolysaccharide (LPS) at $10 \mu \mathrm{g} \cdot \mathrm{mL}^{-1}$ for $20 \mathrm{~h}$. a) Abundance of IL-22R1 mRNA was determined by reverse transcriptase quantitative PCR and normalised to the abundance of HPRT1 mRNA; b) cell surface expression of IL-22R1 protein was assessed using flow cytometry and expressed as the median value of fluorescence; c) cell activation by each stimulus was confirmed by measuring the release of IL- 6 using ELISA; d) BALB/c mice ( $n=5-7$ per group) were infected with a sublethal dose of H3N2 IAV $\left(3 \times 10^{3}\right.$ plaque-forming units). Mouse lungs were collected 2 days post-infection and the abundance of $I L-22 R 1 \mathrm{mRNA}$ was determined using reverse transcriptase quantitative PCR and normalised to that of RPLPO mRNA. Data are pesented as mean \pm SEM of at least three independent experiments. NS: nonsignificant. ${ }^{*}$ : $\mathrm{p}<0.05$, Mann-Whitney test or Kruskal-Wallis test.

multifactorial pathological process and thus cannot be considered solely as an outcome of exposure to cigarette smoke. We examined lung tissue from 129 patients (mean \pm SEM age $65.6 \pm 1$ years) undergoing surgery for lung carcinoma, consisting of 14 (11\%) nonsmokers, 53 (41\%) "healthy" smokers and 62 (48\%) COPD patients. Among the COPD patients, 24 (19\%) were Global Initiative for Chronic Obstructive Lung Disease (GOLD) stage 1, 30 (23\%) were GOLD stage 2 and 8 (6\%) were GOLD stage 3. IL-22R1 expression was neither associated with smoking status nor COPD severity grade (fig. 2c). We obtained similar findings with proximal primary airway epithelial cells from control individuals $(n=6)$, "healthy" smokers $(n=3)$ and COPD patients $(n=4)$ ( $p>0.05$; fig. $2 d)$.

\section{Effect of neutrophil serine proteinases on IL-22R expression}

In view of the foregoing negative data and the evidence that neutrophilia is a pathological hallmark of COPD [17], we then formulated a distinct hypothesis. Can neutrophils impair directly or indirectly IL-22R expression and function? We first examined the effect of supernatant from activated neutrophils on the abundance of IL-22R1 at the surface of IAV-challenged epithelial BEAS-2B cells. IL-22R1 expression was strongly impaired under these conditions, whereas $\alpha$-1-proteinase inhibitor (a broad-spectrum inhibitor of serine proteases including NE, proteinase 3 and CG) prevented the disappearance of the receptor (fig. 3a). To identify the key proteinase involved in the decrease of IL-22R expression, we repeated this experiment with a more specific inhibitor, ACT, which reacts only with CG [18]. Interestingly, ACT prevented the disappearance of IL-22R1 signal, suggesting a prominent role for CG in this process (fig. 3a). Next, we investigated whether IL-22R1 expression was altered in the lungs of a mouse model of cigarette smoke-induced COPD. As expected, the number of neutrophils in fluid collected by BAL was higher in mice chronically challenged by cigarette smoke than in room-air control mice $(>30$-fold higher, $\mathrm{p}<0.01$; fig. 3b). Although the abundance of $I L-22 R 1$ RNA was not affected by cigarette smoke, the abundance of IL-22R1 protein was approximatively twofold lower in the lungs of cigarette smoke-exposed mice compared to control animals $(\mathrm{p}<0.02$; fig. $3 \mathrm{~b})$. 

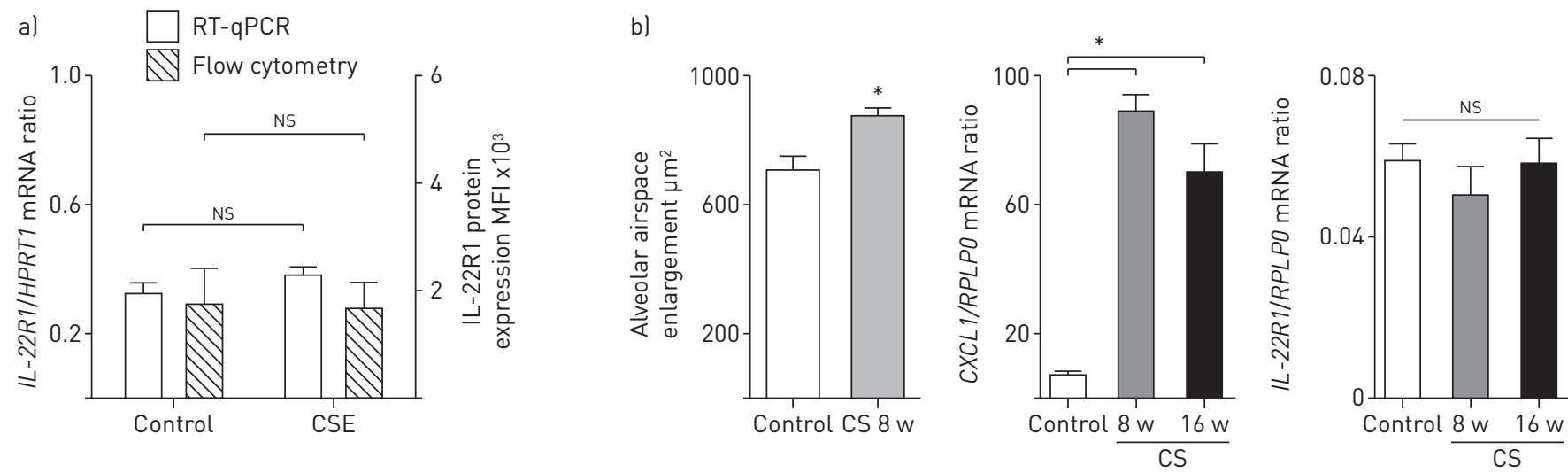

c)

NS

d)
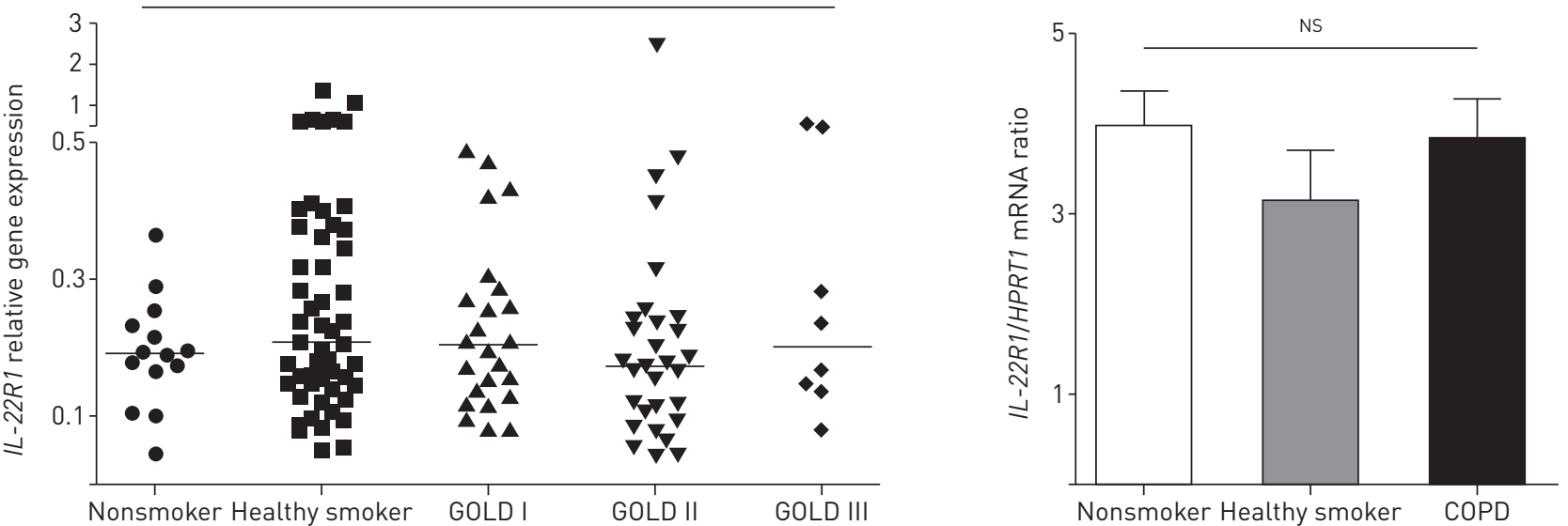

FIGURE 2 Cigarette smoke does not modulate interleukin (IL)-22 receptor (IL-22R) expression. a) Human bronchial epithelial BEAS-2B cells were exposed to 5\% cigarette smoke extract (CSE) or 5\% room air (control) for $6 \mathrm{~h}$. Cell activation by CSE was verified by measuring IL- 8 secretion using ELISA and the expression of $\mathrm{NAD}(\mathrm{P}) \mathrm{H}$ quinone oxidoreductase 1 (NQO1) using reverse transcriptase quantitative (RT-q)PCR (data not shown). The abundance of $I L-22 R 1 \mathrm{mRNA}$ and protein expression was further assessed using RT-qPCR and flow cytometry, respectively. b) BALB/c mice ( $\mathrm{n}=6-10$ per group) were exposed to cigarette smoke (CS) for 8-16 weeks (w) using a whole-body smoke exposure system. The alveolar airspace enlargement in lung sections was measured as an index of emphysema induction; the expression of the inflammatory marker CXCL1 as well as that of $I L-22 R 1$ was analysed in mouse lungs using RT-qPCR. c) $I L-22 R 1$ expression was also assessed using RT-qPCR in human lung tissue samples from 129 patients undergoing thoracic surgery, including 14 (11\%) nonsmokers, 53 (41\%) "healthy" smokers and $62(48 \%)$ chronic obstructive pulmonary disease (COPD) patients. Among the COPD patients, 24 (19\%) were classified as Global Initiative for Chronic Obstructive Lung Disease (GOLD) stage I, 30 (23\%) were GOLD stage II and 8 (6\%) were GOLD stage III. d) IL-22R1 expression was also assessed using RT-qPCR in primary cultures of proximal airway epithelial cells isolated from nonsmokers $(n=6)$, "healthy" smokers $(n=3)$ or COPD patients ( $n=4)$. Data are presented as mean $\pm \operatorname{SEM}\left(\mathrm{a}, \mathrm{b}\right.$ and $\mathrm{d}$ ) of three (a) or two (b) independent experiments. The median is shown in $\mathrm{c}$ ). MFI: mean fluorescence intensity. ${ }^{*}$ : $\mathrm{p}<0.05$ a) Mann-Whitney test or b, c) Kruskal-Wallis test (and Dunn's test for post hoc comparisons).

CG impairs the IL-22-triggered activation of bronchial epithelial cells

Binding of IL-22 to its receptor is known to induce a downstream signalling pathway that involves the phosphorylation at Tyr705 of the transcription factor STAT3. Phosphorylated STAT3 then mediates the biological effects of IL-22 on epithelial cells, such as the production of the antimicrobial peptide $\beta$-defensin 2 [2]. We pretreated bronchial epithelial BEAS-2B cells with either a supernatant of activated neutrophils, or $1 \mu \mathrm{M}$ CG or NE for $30 \mathrm{~min}$ before the addition of an optimal concentration of recombinant IL-22 $\left(20 \mathrm{ng} \cdot \mathrm{mL}^{-1}\right)$ to assess the functional effect of neutrophil serine proteinases on signalling via IL-22R1. The abundance of phosphorylated STAT3 was lower in BEAS-2B cells pretreated with a supernatant of activated neutrophils or NE, but was even more decreased in BEAS-2B cells pretreated with CG than in untreated control cells (fig. 4a). Consistently, the induction of $h B D-2$ expression was also strongly impaired in CG-pretreated cells (fig. 4b).

\section{Cathepsin G cleaves the IL-22R1 and releases a soluble fragment}

Based on the foregoing data which suggested a critical downregulatory effect of CG on IL-22R alteration, we decided to further focus on CG. We found that a progressive decrease of IL-22R1 expression in BEAS-2B cells was negatively correlated with CG concentrations $(0.01-1 \mu \mathrm{M})$, down to $50 \%$ of the control values (fig. $5 \mathrm{a}$ ). We also treated BEAS-2B cells with cytochalasin B before CG treatment to exclude the 

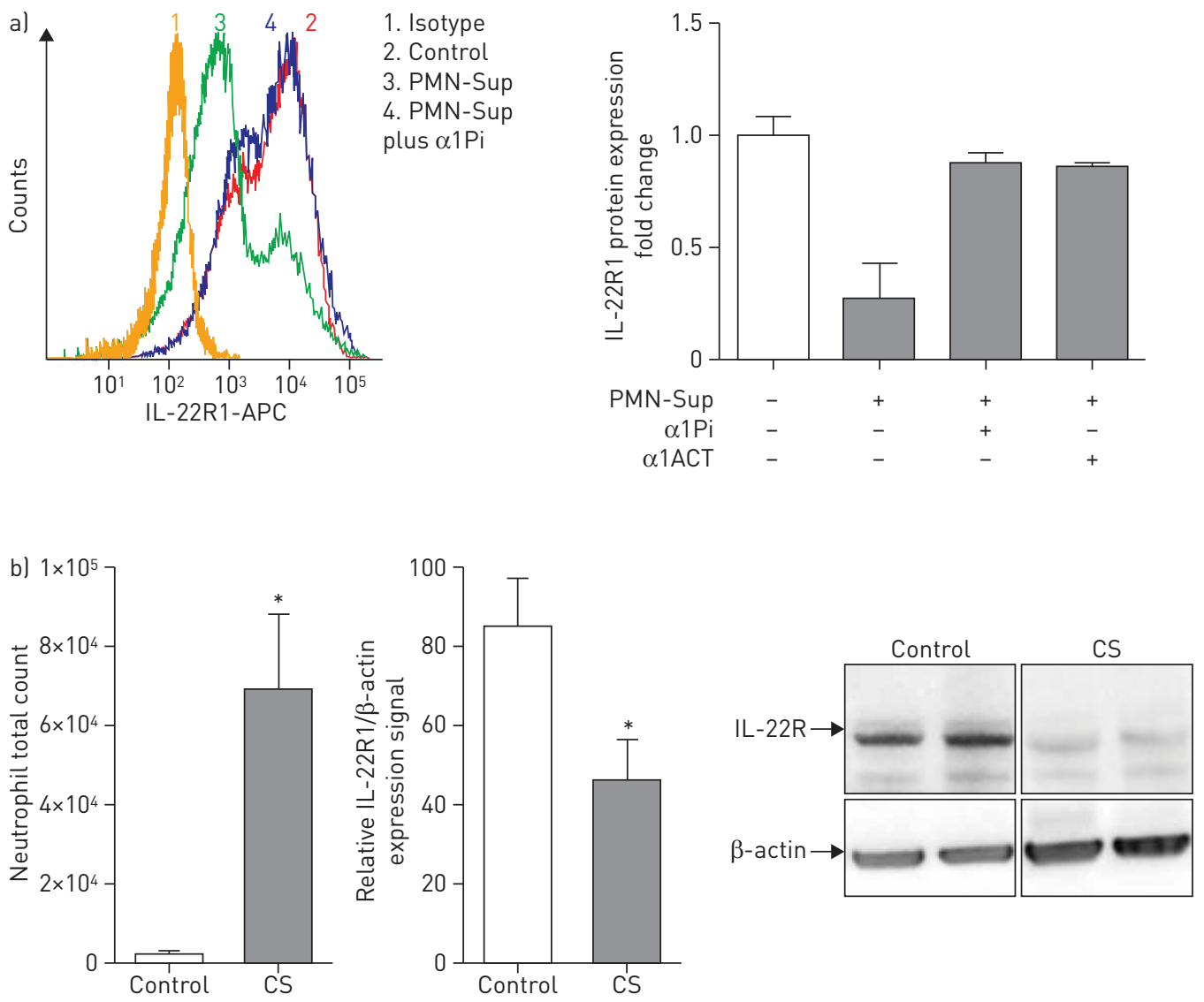

FIGURE 3 Effect of neutrophil serine proteinases on interleukin (IL)-22 receptor (IL-22R) expression. a) Cell surface expression of IL-22R was assessed using flow cytometry in BEAS-2B cells infected with influenza A virus at multiplicity of infection of 1 for $20 \mathrm{~h}$. Cells were further incubated with supernatants from activated neutrophils (PMN-Sup) for $30 \mathrm{~min}$ at $37^{\circ} \mathrm{C}$. $\alpha 1$-protease inhibitor $(\alpha 1 \mathrm{Pi})$ was used to broadly inhibit neutrophil serine proteases (including elastase, proteinase 3 and cathepsin G). $\alpha 1$-antichymotrypsin (ACT) was used to specifically inhibit cathepsin G. b) BALB/c mice ( $\mathrm{n}=6-10$ per group) were exposed for 8 weeks to cigarette smoke (CS) using a whole-body smoke exposure system. Bronchoalveolar lavage fluids were collected and neutrophil counts were assessed using flow cytometry, and the abundance of IL-22R1 protein was determined in the lungs by immunoblotting. The IL-22R1 signal was normalised to that of $\beta$-actin. The results of representative gels are shown. Data are presented as mean \pm SEM of three (a) or two (b) independent experiments. *: $\mathrm{p}<0.05$, Kruskal-Wallis test (and Dunn's test for post hoc comparisons).

possibility that an intracellular pathway triggered by CG results in the downregulation of IL-22R1. The accumulation of this receptor at the cell surface was still strongly impaired under these conditions and was comparable to that observed in cells without cytochalasin B and treated with CG (data not shown). The foregoing results led us to examine whether CG affects the abundance of IL-22R1 by a direct proteolysis. We preformed immunoblotting with cell lysates and supernatants collected from BEAS-2B cells exposed to either a supernatant of activated neutrophils or CG and probed the resulting membranes with a specific antibody targeted against the extracellular part of IL-22R1. We observed a major band of $\sim 62 \mathrm{kDa}$ corresponding to intact IL-22R1 in cell lysates (fig. 5b). By contrast, full-length IL-22R1 was clearly decreased in cells exposed to neutrophil supernatants and undetectable in cells exposed to CG. Conversely, IL-22R1 degradation by CG was prevented by the presence of ACT or when CG was heat-inactivated (fig. 5b). Remarkably, a major band of $\sim 25 \mathrm{kDa}$ was present in the medium of cells exposed to neutrophil supernatants or CG (fig. 5b), suggesting that CG cleaves the IL-22R1 subunit and releases the extracellular part of the receptor into the medium. Next, we sought to investigate whether IL-22R proteolysis also occurs in vivo. Mice were challenged intranasally with purified CG $(0.2 \mu \mathrm{M})$, and BAL fluids were collected $2 \mathrm{~h}$ later for immunoblotting. Neither spontaneous mortality nor lung inflammation occurred under these conditions and IL-6 concentration was similar in BAL fluid from CG-treated and control mice (data not shown). As expected, CG activity was recovered in BAL fluids (fig. 5c). More importantly, a $25-\mathrm{kDa}$ fragment of IL-22R was observed in BAL fluids from CG-exposed mice $(\mathrm{p}<0.02)$, but not in animals which received both CG and $\alpha 1$-ACT (fig. 5 c). 


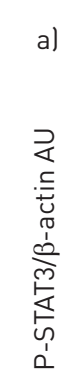
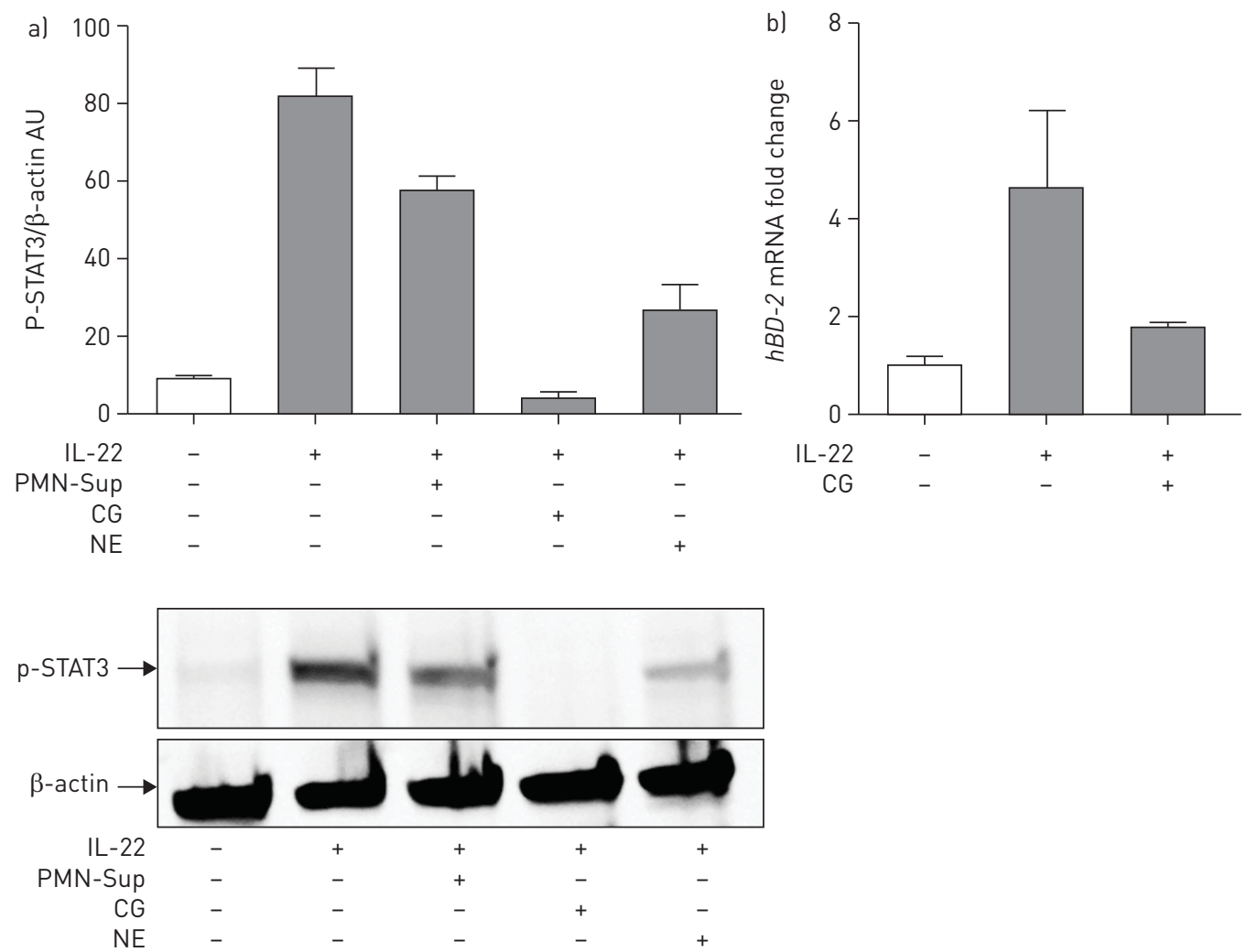

FIGURE 4 a) Functional effect of cathepsin G on interleukin (IL)-22 and IL-22 receptor signalling. BEAS-2B cells were first exposed to either a supernatant from activated neutrophils (PMN-Sup) or $1 \mu \mathrm{M}$ of cathepsin G (CG) or neutrophil elastase (NE) (or were left untreated) for $30 \mathrm{~min}$ at $37^{\circ} \mathrm{C}$ before the addition or not of recombinant IL-22 $\left(20 \mathrm{ng} \cdot \mathrm{mL}^{-1}\right)$. The abundance of the serine-phosphorylated, active form of the transcription factor STAT3 (p-STAT3) was analysed using Western blotting. The p-STAT3 signal was normalised to that of $\beta$-actin. b) The abundance of human $\beta$-defensin $(h B D)$ - 2 mRNA was determined by reverse transcriptase quantitative PCR (normalised to the amount of Hprt1 mRNA) and presented as fold change over negative control. Data are presented as the mean \pm SEM of three independent experiments. AU: arbitrary units.

\section{IL-22R is fragmented during IAV-triggered acute pneumonia}

Next, we investigated whether the soluble fragment of IL-22R1 could be detected in a more pathophysiological context, such as lung infection due to IAV. We hypothesised that IL-22R1 is cleaved by serine proteases released in situ from activated neutrophils, because large numbers of these leukocytes are present in lung tissues infected with IAV [19]. Mice were infected with a sublethal dose of IAV and body weight was monitored daily after infection (fig. 6a). BAL fluids were collected at days 2 and 6 post-infection to assess neutrophil counts and examine IL-22R1 by immunoblotting. Body weight was normal at day 2, and a small number of neutrophils were recruited into lung tissues (fig. 6b). By contrast, at day 6 , mice had lost $\sim 20 \%$ of their initial body weight and their lungs were infiltrated by large numbers of neutrophils (total count $3.7 \times 10^{5}$ cells in BAL fluid, $\mathrm{p}<0.001$ versus control animals). Immunoblotting revealed the presence of a $25-\mathrm{kDa}$ fragment of IL-22R in the airspaces of IAV-infected mice at day 6 post-infection (fig. 6c). Of note, there was a positive relationship between neutrophil count and the abundance of the soluble IL-22R1 fragment in BAL fluids ( $r=0.53, p<0.01$, Spearman test).

\section{A fragment of IL-22R is released into the lungs of AE-COPD patients}

To verify the relevance of our experimental findings in human pathophysiology, we eventually searched for the release of a $25-\mathrm{kDa}$ fragment of IL-22R in sputa from COPD $(n=14)$ or AE-COPD patients $(n=11)$. As expected, the number of neutrophils in sputa was higher in AE-COPD patients than in sputa from COPD patients (fig. 7a, $\mathrm{p}<0.001$ ). Interestingly, we detected a soluble IL-22R1 fragment of $25 \mathrm{kDa}$ in sputa from both COPD and AE-COPD patients (fig. 7b), but the abundance of this fragment was highest in fluids from AE-COPD patients $(\mathrm{p}<0.02)$.

\section{Discussion}

The persistent presence of neutrophils in the lungs of COPD patients is thought to maintain inflammation through the release of active mediators such as proteolytic enzymes [5-7]. Paradoxically, neutrophils 


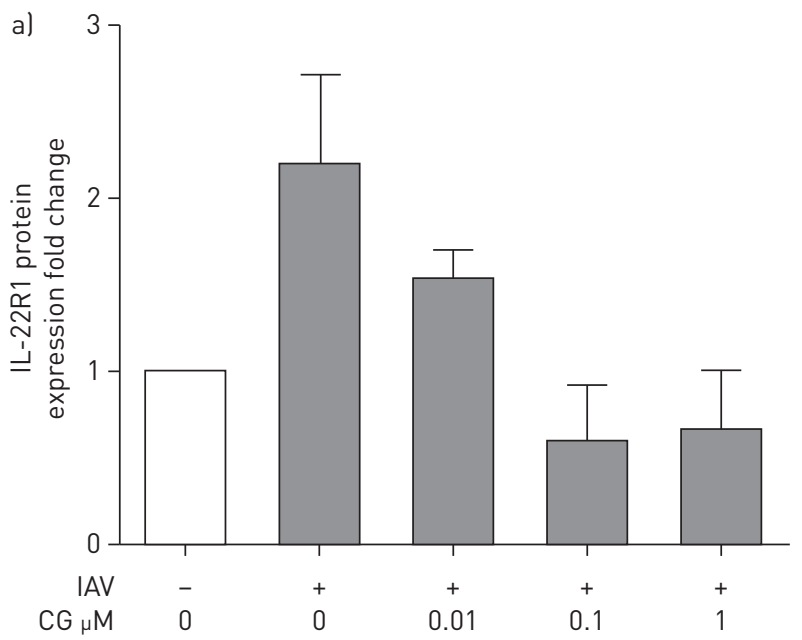

b)
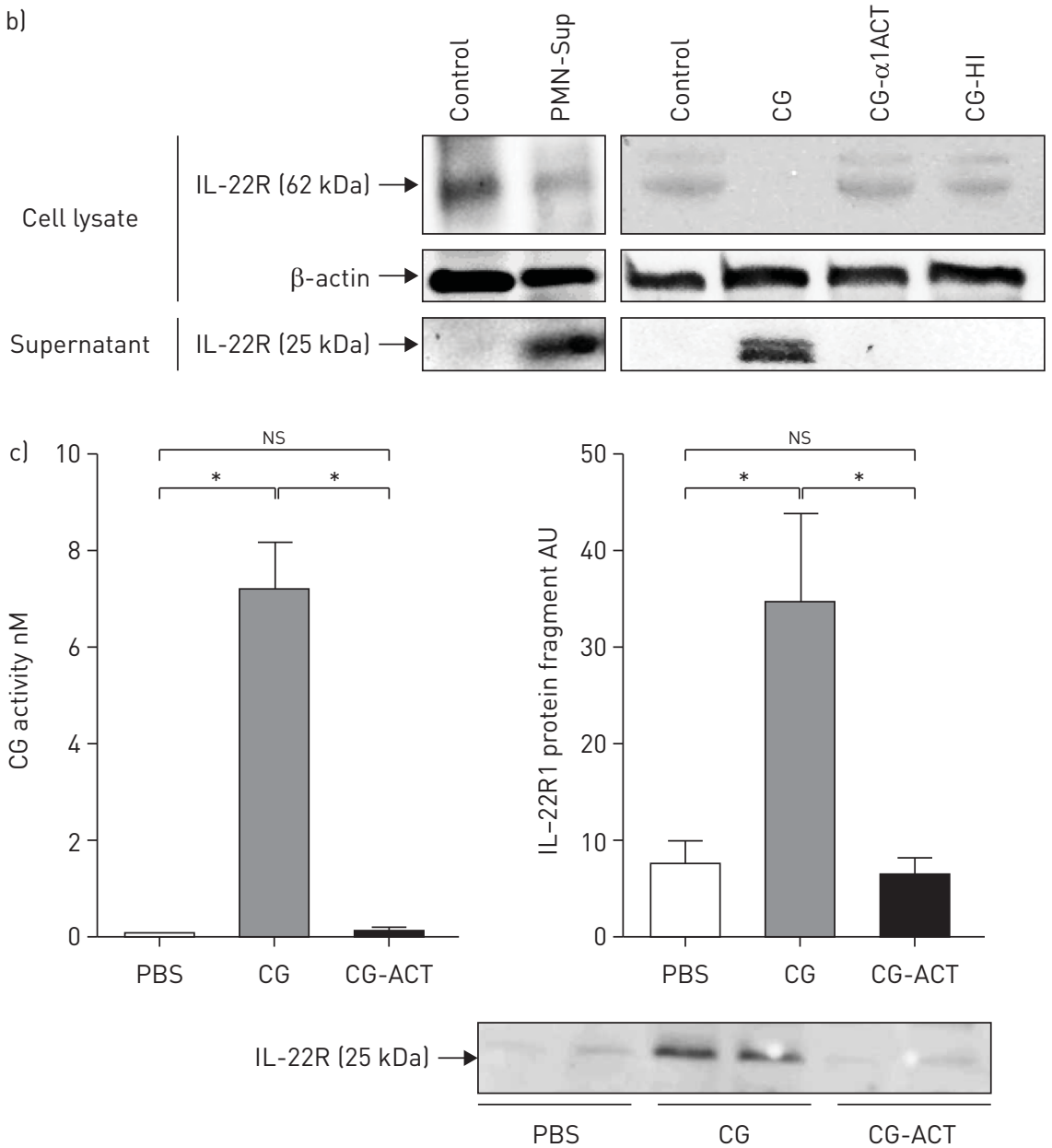

FIGURE 5 Cathepsin G (CG) cleaves interleukin-22 receptor (IL-22R) and releases a 25-kDa soluble fragment. a) Cell surface expression of IL-22R was assessed using flow cytometry in BEAS-2B cells infected with influenza A virus (IAV) at multiplicity of infection of 1 for $20 \mathrm{~h}$. Cells were further incubated with increasing concentrations of CG $(0.01-1 \mu \mathrm{M})$ for $30 \mathrm{~min}$ at $37^{\circ} \mathrm{C}$. b) The abundance of IL-22R in BEAS-2B cells exposed to either a supernatant of activated neutrophils (PMN-Sup) or to $1 \mu \mathrm{M}$ of CG for $30 \mathrm{~min}$ at $37^{\circ} \mathrm{C}$ was assessed using Western blotting in cell lysates and in the corresponding cell supernatants. BEAS-2B cells were also exposed to CG previously inhibited by $\alpha 1$-antichymotrypsin (ACT) or heat-inactivated (HI) to obtain non-enzymatically active CG. c) BALB/c mice were intranasally challenged with $40 \mu \mathrm{L}$ of CG $(0.2 \mu \mathrm{M})$, PBS or ACT-treated CG and bronchoalveolar lavage fluids were collected $2 \mathrm{~h}$ later. CG enzymatic activity and the abundance of IL-22R protein were further determined. The results of one representative gel (out of three) are shown. Data are presented as mean \pm SEM of three independents experiments. NS: nonsignificant; AU: arbitrary units. ${ }^{*}$ : $\mathrm{p}<0.05$, Kruskal-Wallis test (and Dunn’s test for post hoc comparisons). 


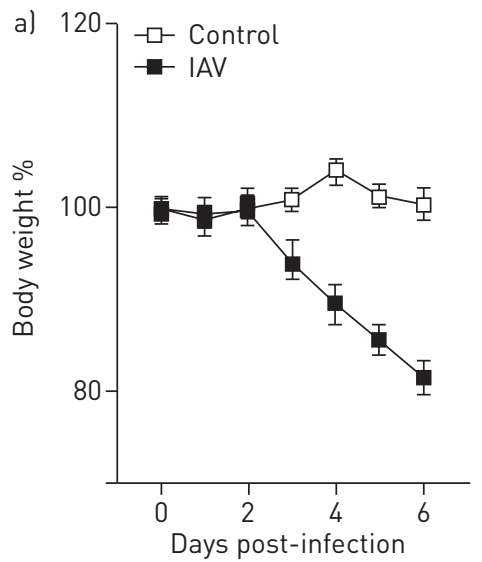

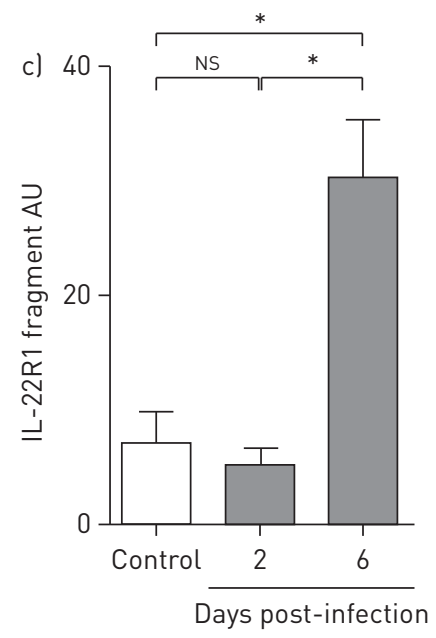

IL-22R1 (25 kDa)

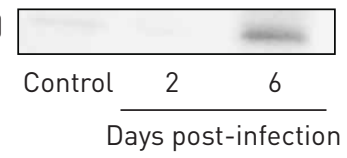

FIGURE 6 Interleukin-22 receptor (IL-22R) is fragmented in mouse lungs during infection-driven inflammation. a) BALB/c mice were infected with a sublethal dose of influenza A virus (IAV). Body weight was monitored daily after infection. At days 2 or 6 post-infection, bronchoalveolar lavage fluids were collected to b) assess neutrophil counts using flow cytometry and c) to examine IL-22R1 expression by immunoblotting; results of one representative gel are shown. Ns: nonsignificant; AU: arbitrary units. Data are presented as mean \pm SEM ( $n=6$ mice per group) and are representative of two independent experiments. ${ }^{*}$ : $p<0.05$, Kruskal-Wallis test (and Dunn's test for post hoc comparisons).

present in the airway mucosa of COPD patients should theoretically play a key role in antimicrobial defence. On the contrary, high numbers of neutrophils recruited to the lungs positively correlate with poor prognosis [20]. Besides, the clinical course of COPD is punctuated by infection-driven acute exacerbations $[5,6,21]$. Thus, an understanding of the potential adverse effects of neutrophils on lung antimicrobial defence may provide insight for the development of new therapies to limit AE-COPD and attenuate the disease [22]. Here, we show for the first time that neutrophil proteases cleave the IL-22 receptor expressed in the lung mucosa and we reveal the harmful effect of this event on antimicrobial responses.

IL-22/IL-22R signalling is pivotal at barrier surfaces where epithelial cells play an active role in the initiation, regulation and resolution of immune responses. Functional studies in mouse models indicate
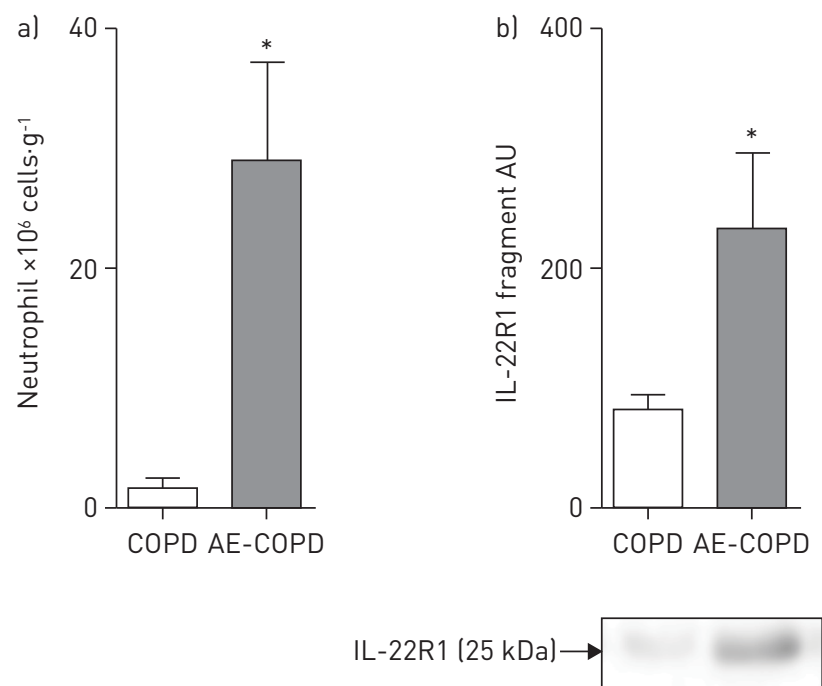

FIGURE 7 Interleukin-22 receptor (IL-22R) is significantly cleaved in the lungs of patients with acute exacerbations (AE) of chronic obstructive pulmonary disease (COPD). Sputa from COPD patients with $(n=11)$ or without $(n=14)$ acute exacerbations were collected. a) Neutrophil counts were assessed using flow cytometry; b) IL-22R1 protein expression was examined using immunoblotting. A pool of sputum served as calibrator for comparisons. Data are presented as mean \pm SEM. AU: arbitrary units. *: $\mathrm{p}<0.05$, Mann-Whitney test. 
that IL-22 has immunoregulatory properties in infection, inflammation, autoimmunity and cancer $[2,3]$. Studies involving genetic or antibody-mediated disruption of the IL-22/IL-22R pathway in mice suggest that this pathway plays an essential role in antimicrobial defence. For instance, IL-22-deficient mice infected with Klebsiella pneumoniae in the lungs or Citrobacter rodentium in the intestine die more rapidly than infected wild-type animals. The protective effects of IL-22 mostly involve the production of antimicrobial peptides. Moreover, IL-22 promotes maintenance and repair of the epithelial barrier in the intestine and respiratory tract, thus limiting pathogen dissemination $[2,3]$. We and others have shown also that IL-22 is required for lung defence and repair after infection with influenza virus [23] or after co-infection with influenza and Streptococcus pneumoniae [4]. Hence IL-22 is critical for mucosal immunity against viral and bacterial infections.

IL-22/IL-22R-dependent signalling involves predominantly the transcription factor STAT3. STAT3 deficiency in epithelial cells mimics that of IL-22 in a mouse model of colitis, suggesting that STAT3 is required for IL-22 dependent signalling in vivo [24]. Consistently, IL-22R1 cleavage by CG strongly inhibited STAT3-dependent signalling and $h B D-2$ expression. Given these data, and the observation that the excessive secretion of neutrophil proteases is positively correlated with COPD severity [5, 6], we believe that our current findings are highly relevant to the pathogenesis of AE-COPD. Accordingly, we propose a mechanism based on previous findings and our current findings $[2,3,5,6]$ to explain how neutrophil proteases may predispose COPD patients to life-threatening infections (fig. 8). 1) Cigarette smoke in the lungs contributes to neutrophil infiltration and degranulation, resulting in the release of active proteases; 2) Matrix components and soluble mediators are degraded, which generates products acting as pro-inflammatory stimuli in a feedback loop [10]; 3) CG and other neutrophil proteases degrade IL-22R and impair the downstream STAT3-dependent antimicrobial effectors; and 4) such protease-dependent disarming of the IL-22/IL-22R axis may facilitate the replication and spread of pathogens, especially bacteria [4] and consequently, may be detrimental in the setting of AE-COPD.

It is noteworthy that the IL-22R1 subunit interacts with two other members of the IL-10 cytokine family in addition to IL-22, IL-20 and IL-24 [25]. IL-20 is thought to amplify the actions of IL-22 through a

a)

$$
\text { Prevention of lung infection }
$$

$\Delta$

Expression of antimicrobial peptides Tissue repair

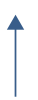

Functional IL-22R pathway

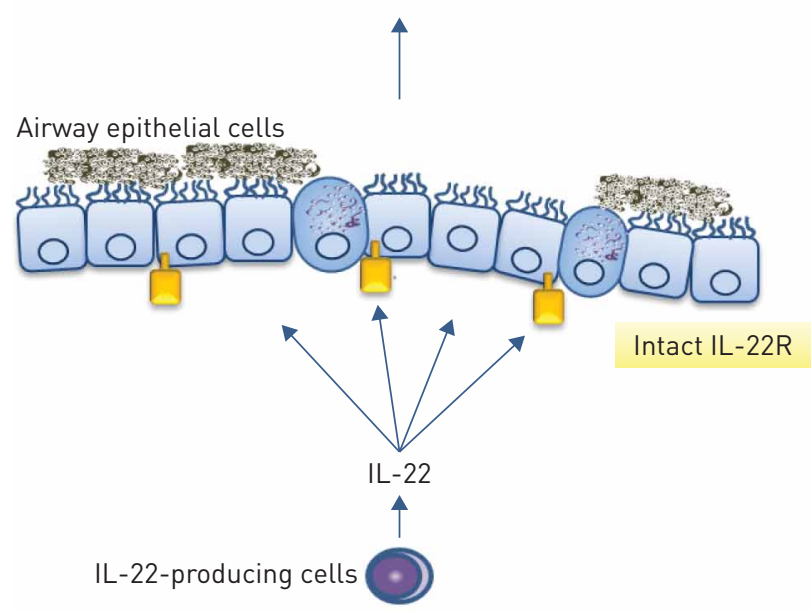

b)

Susceptibility to lung infection
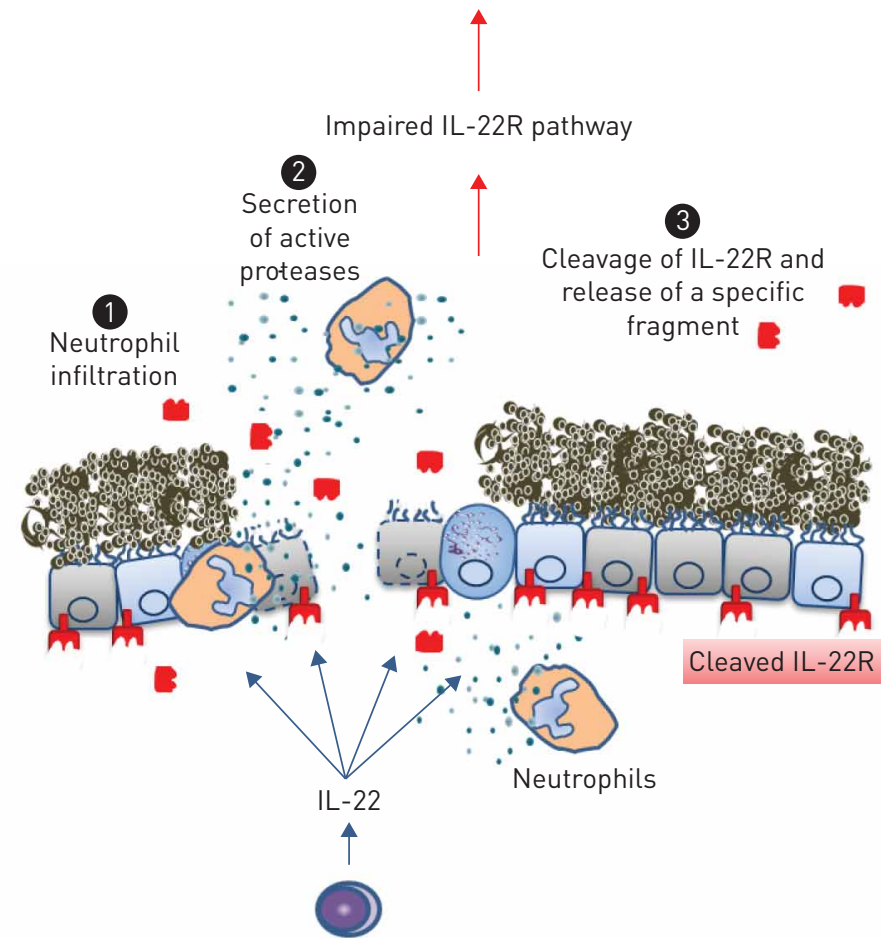

FIGURE 8 Model of alterations of the interleukin (IL)-22/IL-22 receptor (IL-22R) pathway in chronic obstructive pulmonary disease (COPD). a) In healthy lung mucosa, IL-22 stimulates the production of antimicrobial peptides and promotes the maintenance and repair of the respiratory epithelial barrier, thus limiting pathogen burden and dissemination. IL-22 mediates these effects via the IL-22R expressed at the surface of airway epithelial cells. b) Neutrophil infiltration is a prominent feature of COPD lungs (1) and neutrophils from patients with COPD are primed, resulting in the release of active proteases (2). Neutrophil-derived proteases cleave the IL-22R (3) and inhibit downstream STAT3-dependent antimicrobial signalling. This major alteration of the immune response of the lung mucosa may predispose COPD patients to infection-triggered exacerbations. 
positive feedback loop [26, 27]. IL-24 appears to be antiproliferative in the context of wound healing and also protects against bacterial infections [28]. Thus, it is tempting to speculate that cells bearing IL-22R become refractory not only to IL-22 but also to IL-20 and IL-24, following IL-22R1 cleavage by CG in the lungs of COPD patients, conferring an unanticipated immunoregulatory activity of neutrophil proteases.

\section{Conclusions}

As in all chronic lung inflammatory diseases, COPD is associated with episodes of acute exacerbations, which are essentially the result of viral and bacterial infections. Despite the life-threatening nature of these exacerbations, the mechanisms underlying them are still unclear. Here, we show that neutrophil-derived proteases may contribute to AE-COPD by impairing the antimicrobial IL-22/IL-22R signalling pathway. Accordingly, our findings support the renewed interest in neutrophil-derived proteases as key therapeutic targets in COPD $[6,29]$.

\section{Acknowledgements}

The authors thank the patients who volunteered for this study. They also appreciate the help of Veronique Siméon, Christine Mabilat and Aurélie Aubrey (University Hospital of Tours, Tours, France) for the collection of human samples. They also thank Virginie Thibaut, Amandine Vallet, Benjamin Plante and Chrystophe Aubert (Centre d'Etude des Pathologies Respiratoires and University of Tours) for their technical support and Reuben Ramphal (University of Florida, Gainesville, FL, USA) for his advice during manuscript preparation.

\section{References}

1 Wedzicha JA, Seemungal TAR. COPD exacerbations: defining their cause and prevention. Lancet 2007; 370: 786-796.

2 Sabat R, Ouyang W, Wolk K. Therapeutic opportunities of the IL-22-IL-22R1 system. Nat Rev Drug Discov 2013 13: $21-38$.

3 Sonnenberg GF, Fouser LA, Artis D. Functional biology of the IL-22-IL-22R pathway in regulating immunity and inflammation at barrier surfaces. Adv Immunol 2010; 107: 1-29.

4 Ivanov S, Renneson J, Fontaine J, et al. Interleukin-22 reduces lung inflammation during influenza A virus infection and protects against secondary bacterial infection. J Virol 2013; 87: 6911-6924.

5 Hoenderdos K, Condliffe A. The neutrophil in chronic obstructive pulmonary disease. Am J Respir Cell Mol Biol 2013; 48: 531-539.

6 Meijer M, Rijkers GT, van Overveld FJ. Neutrophils and emerging targets for treatment in chronic obstructive pulmonary disease. Expert Rev Clin Immunol 2013; 9: 1055-1068.

7 Stockley RA. Alpha1-antitrypsin review. Clin Chest Med 2014; 35: 39-50.

8 Le-Barillec K, Pidard D, Balloy V, et al. Human neutrophil cathepsin G down-regulates LPS-mediated monocyte activation through CD14 proteolysis. J Leukoc Biol 2000; 68: 209-215.

9 Le-Barillec K, Si-Tahar M, Balloy V, et al. Proteolysis of monocyte CD14 by human leukocyte elastase inhibits lipopolysaccharide-mediated cell activation. J Clin Invest 1999; 103: 1039-1046.

10 Pham CT. Neutrophil serine proteases: specific regulators of inflammation. Nat Rev Immunol 2006; 6: 541-550

11 Chignard M, Pidard D. Neutrophil and pathogen proteinases versus proteinase-activated receptor-2 lung epithelial cells: more terminators than activators. Am J Respir Cell Mol Biol 2006; 34: 394-398.

12 Guillot L, Le Goffic R, Bloch S, et al. Involvement of toll-like receptor 3 in the immune response of lung epithelial cells to double-stranded RNA and influenza A virus. J Biol Chem 2005; 280: 5571-5580.

13 Jyot J, Balloy V, Jouvion G, et al. Type II secretion system of Pseudomonas aeruginosa: in vivo evidence of a significant role in death due to lung infection. J Infect Dis 2011; 203: 1369-1377.

14 Dubois AV, Gauthier A, Bréa D, et al. Influence of DNA on the activities and inhibition of neutrophil serine proteases in cystic fibrosis sputum. Am J Respir Cell Mol Biol 2012; 47: 80-86.

15 Korkmaz B, Attucci S, Juliano MA, et al. Measuring elastase, proteinase 3 and cathepsin G activities at the surface of human neutrophils with fluorescence resonance energy transfer substrates. Nat Protoc 2008; 3: 991-1000.

16 Brusselle GG, Joos GF, Bracke KR. New insights into the immunology of chronic obstructive pulmonary disease. Lancet 2011; 378: 1015-1026.

17 Sethi S, Maloney J, Grove L, et al. Airway inflammation and bronchial bacterial colonization in chronic obstructive pulmonary disease. Am J Respir Crit Care Med 2006; 173: 991-998.

18 Duranton J, Boudier C, Belorgey D, et al. DNA strongly impairs the inhibition of cathepsin G by $\alpha_{1}$-antichymotrypsin and $\alpha_{1}$-proteinase inhibitor. J Biol Chem 2000; 275: 3787-3792.

19 Si-Tahar M, Blanc F, Furio L, et al. Protective role of LGP2 in influenza virus pathogenesis. J Infect Dis 2014; 210: 214-223.

20 Gernez Y, Tirouvanziam R, Chanez P. Neutrophils in chronic inflammatory airway diseases: can we target them and how? Eur Respir J 2010; 35: 467-469.

21 Donaldson GC, Seemungal TAR, Bhowmik A, et al. Relationship between exacerbation frequency and lung function decline in chronic obstructive pulmonary disease. Thorax 2002; 57: 847-852.

22 Bauer CMT, Morissette MC, Stämpfli MR. The influence of cigarette smoking on viral infections: translating bench science to impact COPD pathogenesis and acute exacerbations of COPD clinically. Chest 2013; 143: 196-206.

23 Pociask DA, Scheller EV, Mandalapu S, et al. IL-22 is essential for lung epithelial repair following influenza infection. Am J Pathol 2013; 182: 1286-1296.

24 Pickert G, Neufert C, Leppkes M, et al. STAT3 links IL-22 signaling in intestinal epithelial cells to mucosal wound healing. J Exp Med 2009; 206: 1465-1472.

25 Dumoutier L, Leemans C, Lejeune D, et al. Cutting edge: STAT activation by IL-19, IL-20 and mda-7 through IL-20 receptor complexes of two types. J Immunol 2001; 167: 3545-3549. 
26 Wolk K, Haugen HS, Xu W, et al. IL-22 and IL-20 are key mediators of the epidermal alterations in psoriasis while IL-17 and IFN- $\gamma$ are not. J Mol Med 2009; 87: 523-536.

27 Wolk K, Witte E, Warszawska K, et al. The Th17 cytokine IL-22 induces IL-20 production in keratinocytes: a novel immunological cascade with potential relevance in psoriasis. Eur J Immunol 2009; 39: 3570-3581.

28 Whitaker EL, Filippov VA, Duerksen-Hughes PJ. Interleukin 24: mechanisms and therapeutic potential of an anti-cancer gene. Cytokine Growth Factor Rev 2012; 23: 323-331.

29 Matera MG, Calzetta L, Segreti A, et al. Emerging drugs for chronic obstructive pulmonary disease. Expert Opin Emerg Drugs 2012; 17: 61-82. 